\title{
Intravesical Mitomycin-C with Bladder Wall Hyperthermia in Intermediate and High-risk Non-muscle Invasive Bladder Cancers: Prospective Clinical Trial with a Single- treatment Arm
}

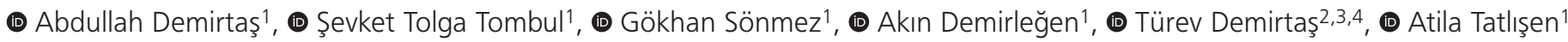 \\ ${ }^{1}$ Erciyes University Faculty of Medicine, Department of Urology, Kayseri, Turkey \\ ${ }^{2}$ Erciyes University Faculty of Medicine, Department of Medical History and Ethics, Kayseri, Turkey \\ ${ }^{3}$ Erciyes University Faculty of Medicine, Department of Stem Cell Sciences, Genom and Stem Cell Center, Kayseri, Turkey \\ ${ }^{4}$ Erciyes University, Halil Bayraktar Vocational Health Collage, Department of Emergency Medical Program, Kayseri, Turkey
}

\begin{abstract}
Objective: Bacillus Calmette-Guérin (BCG) limited availability, cost, and allergic response in some patients limit its use, leading to the search for alternative agents. We aimed to present the outcomes of intermediate- and high-risk non-muscle invasive bladder cancers treated with bladder wall thermochemotherapy (BWT) system via a single-arm prospective study.

Materials and Methods: Overall, 44 patients were enrolled and subjected to a treatment with mitomycin-C and BWT for 6 weeks, followed by a monthly maintenance for up to 1 year. The recurrence rate, progression rate, recurrence-free survival, progression-free survival, and side effects were evaluated.

Results: In the intermediate- and high-risk groups, the median follow-up duration was 14 and 17 months; recurrence rates were $28.6 \%$ and $23.3 \%$; progression rates were $7.1 \%$ and $23.3 \%$; the recurrence free survival at the $24^{\text {th }}$ month were $78.6 \%$ and $80.0 \%$ and the progression free survival at the $24^{\text {th }}$ month were $92.6 \%$ and $76.7 \%$, respectively. All the patients suffered from dysuria lasting for 2-3 days; five of the 442 BWT application developed allergic reactions requiring treatments with oral agents.

Conclusion: BWT showed comparable recurrence and progression rates, but seemed safer than BCG when our findings were compared to those of published studies. It could be an alternative for individuals who are not suitable for BCG or in a case of BCG unavailability.

Keywords: Bladder, cancer, intravesical, mitomycin, thermo
\end{abstract}

\section{Introduction}

Among all cancers, bladder cancer $(\mathrm{BC})$ ranks $10^{\text {th }}$ in incidence among both sexes. At the time of diagnosis, most BCs (70\%-80\%) are non-muscle invasive (NMI) $(1,2)$. It is advisable to stratify them into low-, intermediate-, and high-risk groups based on their clinical features, pathological stages, and grades because NMI-BC patients show varying risks in terms of recurrence and progression. For the low-risk group, one dose of intravesical chemotherapy immediately after transurethral resection of the BC (TURBT) is sufficient in almost all patients. However, for the intermediate- and high-risk groups, it is quite challenging to tailor the appropriate treatment. Although intravesical chemotherapy prevents recurrences, it is not as effective in preventing their progression as intravesical Bacillus CalmetteGuérin (BCG) for the intermediate-risk group. Intravesical BCG with a one-year maintenance was demonstrated to be superior to intravesical chemotherapy in preventing BC progression in the intermediate-risk group. Intravesical BCG with 1-3 years of maintenance or radical cystectomy is another treatment option suggested for the high-risk group (3). However, the fact that BCG has serious side effects and is not always readily available from the market has escalated the search for new alternatives such as intravesical mitomycin-C (MMC) application with 
hyperthermia [thermochemotherapy (TCT)]. Several studies have compared TCT with intravesical chemotherapy alone or with BCG, which employed a microwave technology as the heating system $(4,5,6)$. A limited number of studies have employed a conductive heating system $(7,8)$.

This paper presents the recurrence and progression outcomes of intermediate- and high-risk NMIBC treated with the bladder wall TCT system.

\section{Materials and Methods}

\section{Patient Selection}

The study was designed as a prospective study with a singletreatment arm, including MMC with TCT. The study group included NMIBC patients who were newly diagnosed or followed-up as recurrent patients between January 2014 and February 2015. They were stratified into intermediate and highrisk groups according to the criteria defined in the European Association of Urology Guidelines on "non-muscle invasive urothelial carcinoma of bladder" (3). All of them had been histologically diagnosed with urothelial carcinoma. The exclusion criteria included low bladder capacity $(<150 \mathrm{~mL})$, increased postvoiding residual urine $(>150 \mathrm{~mL})$, untreatable or uncontrollable urinary tract infection, history of urethral stricture, presence of bladder diverticula $>1 \mathrm{~cm}$ size, pathology other than urothelial carcinoma, World Health Organization performance status $>2$, and upper urinary tract urothelial carcinoma diagnosis. Before the application of TCT, complete TURBT was performed. Restaging TURBT (RE-TURBT) was conducted within four weeks for patients with initial T1 pathology. Postoperative single-dose intravesical chemotherapy was applied to all the patients. The first TCT instillation was applied at 1 month following the TURBT or the RE-TURBT.

\section{Thermochemotherapy Application and Treatment Schedule}

The bladder wall TCT (BWT) system (Elmedical, Hod-Hasharon, Israel) was used as a conductive heating modality. MMC (40 mg; MMC Kyowa Hakko Kogyo Co., Ltd., Tokyo, Japan) was mixed with $50 \mathrm{~mL}$ of $0.9 \%$ saline solution and applied using a disposable silicone $20 \mathrm{Fr}$ 3-way TCT catheter (UniThermia catheter; Elmedical). In each session, the bladder was irrigated with $\mathrm{MMC}$ solution at $42{ }^{\circ} \mathrm{C}-45^{\circ} \mathrm{C}$ for 45 minute via the BWT system. The treatment schedule consisted of an initial 6-week instillation, followed by control cystoscopy and urine cytology in the $3^{\text {rd }}$ month and monthly instillations for up to 1 year. Urine cytology and cystoscopy were performed every three months. The samples were collected from the suspected areas during cystoscopy. At the end of the first year, a control cystoscopy with random bladder biopsies was conducted. During the second year, routine controls were conducted every three months. Subsequently, follow-ups were performed every six months. In case of the development of any complication in a patient, TCT was discontinued. Before each instillation of TCT, clear urine cultures were obtained from all the patients. No prophylactic anticholinergic agent was administered prior to the intravesical
TCT. If TURBT pathology was Ta or T1 during the follow-up, it was accepted as a recurrence. If it was carcinoma in situ or T2, it was accepted as a progression. All the adverse events observed during the study were recorded according to the Common Terminology Criteria for Adverse Events (CTCAE) Version 4.0 (9).

All the study protocols were approved by the local ethical committee at our institution on July, 4 ${ }^{\text {th }}, 2014$ (IRB Approval number: 2014/387). An informed consent was obtained from all the patients.

\section{Statistical Analysis}

Statistical analysis was performed using the Statistical Package for the Social Sciences (SPSS) version 22 for windows (IBM, NY, USA) software package. Normality test was done with ShapiroWilks test. Survival analysis was done with Kaplan-Meier tests and survival tables.

\section{Results}

In total, 44 patients (females $=2$, rest were males) were enrolled in this study, of which 14 showed intermediate-risk NMIBC and 30 showed high-risk NMIBC. The patients' characteristics are summarised in Table 1. Eight patients had a history of intravesical chemotherapy or BCG. The median follow-up period was 14 months in the intermediate-risk group and 17 months in the high-risk group. In both groups, the median number of BWT instillation was 9 . The recurrence rate was $28.6 \%(4 / 14)$ in the intermediate-risk group and $23.3 \%$ (7/30) in the high-risk group. Progression was detected in only one $(7.1 \%)$ patients from the intermediate-risk group and seven (23.3\%) patients from the high-risk group. Four patients showed progression to the T2 stage, three of whom received radical cystectomy and the remaining one patient was at the metastatic stage clinically and preferred a bladder-preserving treatment modality (chemoradiotherapy). The progression pathology was carcinoma in situ in the remaining four patients. The initial pathology before TCT was also carcinoma in situ in two of them. Radical cystectomy was suggested to patients with carcinoma in situ after TCT, but they preferred intravesical BCG. In the intermediate- and highrisk groups, the recurrence free survival rates at the $24^{\text {th }}$ month were $78.6 \%$ and $80 \%$ and the progression free survival rates at the $24^{\text {th }}$ month were $92.6 \%$ and $76.7 \%$, respectively. The Kaplan-Meier curves of recurrence-free survival and progressionfree survival are shown in Figures 1 and 2.

After TCT, no patient reported bladder spasms, bladder pain, and urgency or frequency. However, all the patients reported dysuria resulting from catheterisation that lasted for 2-3 days. No action was taken other than the conservative management for dysuria. Overall, 442 TCT applications were conducted. Allergic reactions (skin rash and hyperemia) were observed in five out of the 442 TCT treated patients (1.12\%), who were treated with oral anti-histaminic agents. None of these allergic attacks broke out with the first TCT, but the study protocol was discontinued for these patients. Urethral stricture developed in one patient after the $9^{\text {th }}$ TCT application, upon which TCT was discontinued for them. It was hard to confirm TCT application as the reason for urethral stricture because this patient had BC 


\begin{tabular}{|c|c|c|c|}
\hline & $\begin{array}{l}\text { Intermediate } \\
\mathrm{n}=14\end{array}$ & $\begin{array}{l}\text { High } \\
\mathrm{n}=30\end{array}$ & $\begin{array}{l}\text { Overall } \\
\mathrm{n}=44\end{array}$ \\
\hline \multicolumn{4}{|l|}{ Gender } \\
\hline - Male & $13(92.9 \%)$ & $29(96.7 \%)$ & $42(95.5 \%)$ \\
\hline - Female & $1(7.1 \%)$ & $1(3.3 \%)$ & $2(4.5 \%)$ \\
\hline Age in years & $68.14 \pm 10.75$ & $64.73 \pm 8.74$ & $65.82 \pm 9.43$ \\
\hline \multicolumn{4}{|c|}{ Previous intravesical treatment } \\
\hline - No previous treatment & $12(85.7 \%)$ & $24(80 \%)$ & $36(81.8 \%)$ \\
\hline - MMC & $1(7.1 \%)$ & $1(3.3 \%)$ & $2(4.5 \%)$ \\
\hline - BCG & $1(7.1 \%)$ & $3(10 \%)$ & $4(9.1 \%)$ \\
\hline - $\mathrm{MMC}+\mathrm{BCG}$ & 0 & $2(6.7 \%)$ & $2(4.5 \%)$ \\
\hline \multicolumn{4}{|l|}{ Grade } \\
\hline - Low Grade & 14 & $16(53.3 \%)$ & $30(68.2 \%)$ \\
\hline - High Grade & 0 & $14(46.7 \%)$ & $14(31.8 \%)$ \\
\hline \multicolumn{4}{|l|}{ Stage } \\
\hline • Ta & 14 & $6(20 \%)$ & $20(45.5 \%)$ \\
\hline • $\mathrm{T} 1$ & 0 & $22(73.3 \%)$ & $22(50 \%)$ \\
\hline - CIS & 0 & $2(6.7 \%)$ & $2(4.5 \%)$ \\
\hline $\begin{array}{l}\text { Follow-up in } \\
\text { months -median } \\
\text { (min-max) }\end{array}$ & $14(3-41)$ & $17(2-47)$ & $16.5(2-47)$ \\
\hline $\begin{array}{l}\text { \# of BWT session-median } \\
\text { (min-max) }\end{array}$ & $9(2-16)$ & $9(4-16)$ & $9(2-16)$ \\
\hline Recurrence & $4(28.6 \%)$ & $7(23.3 \%)$ & $11(25 \%)$ \\
\hline Progression & $1(7.1 \%)$ & $7(23.3 \%)$ & $8(18.2 \%)$ \\
\hline \multicolumn{4}{|l|}{ Adverse event } \\
\hline - Allergic reaction & $1(7.1 \%)$ & $4(13.3 \%)$ & $5(11.4 \%)$ \\
\hline - Urethral stricture & 0 & $1(3.3 \%)$ & $1(2.3 \%)$ \\
\hline \multicolumn{4}{|l|}{ Treatment after progression } \\
\hline - Radical cystectomy & $1(7.1 \%)$ & $2(6.7 \%)$ & $3(6.8 \%)$ \\
\hline $\begin{array}{l}\text { - Radiotherapy \& } \\
\text { chemotherapy }\end{array}$ & 0 & $1(3.3 \%)$ & $1(2.3 \%)$ \\
\hline - BCG with maintenance & 0 & $4(13.3 \%)$ & $4(9.1 \%)$ \\
\hline
\end{tabular}

for $>5$ year and a history of intravesical MMC and BCG. All these adverse events were of $\leq$ grade 2 according to CTCAE v4.0.

\section{Discussion}

The search for new agents and administration routes for intravesical treatment to prevent recurrence and progression in NMIBC is actively ongoing. MMC, which is safer than BCG, does not have the same efficacy to prevent progression as it does for recurrence (10).

Accordingly, in an attempt to increase its efficacy, MMC application with hyperthermia has gained much attention for application and research since the past 20 years. Colombo et al. (5) reported recurrence rates of MMC alone and TCT + MMC in a median 90 months of follow-up as $80 \%$ and $14 \%$, respectively, whereas the progression rates were $15 \%$ and $8.6 \%$, respectively.

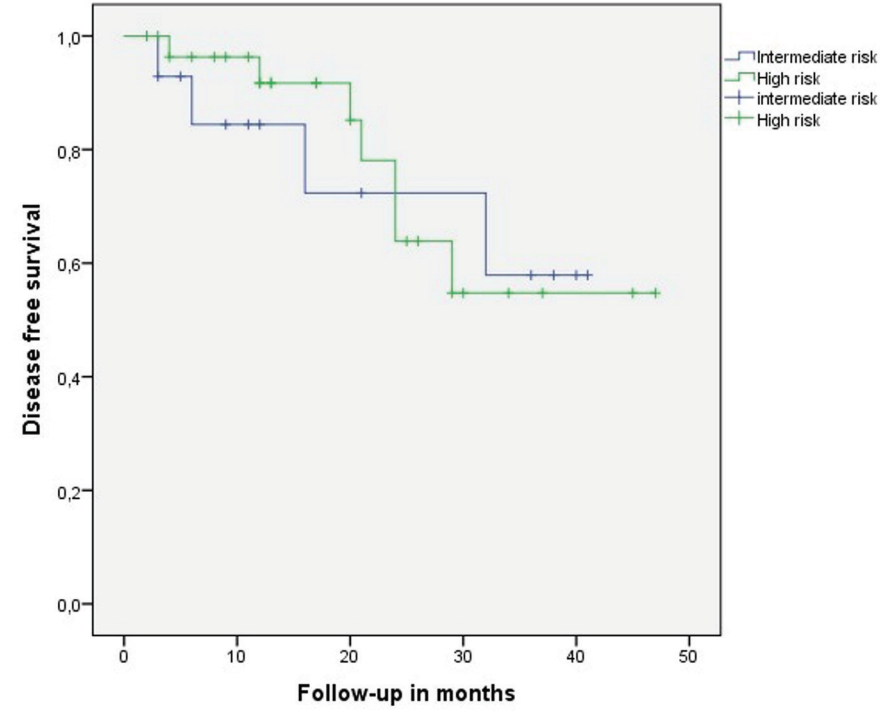

Figure 1. Kaplan-Meier curve of recurrence free survival in both groups

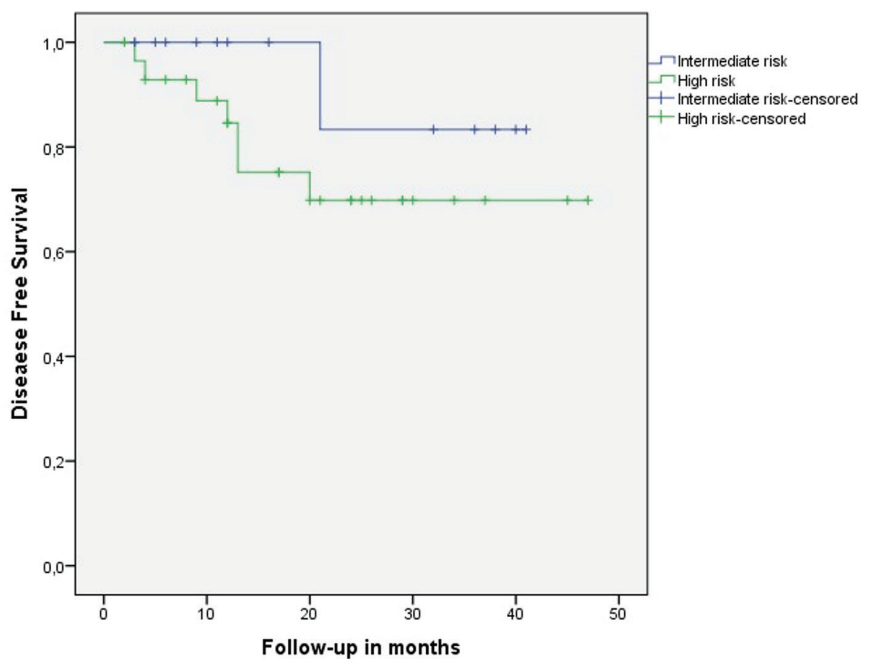

Figure 2. Kaplan-Meier curve of progression free survival in both groups

Another study (4) that used the same hyperthermia system reported a recurrence rate of $9 \%$ in the TCT group at the end of a medianof 289 days of follow-up. In the multicentric phases 1 and 2 study of the conductive heating modality, the recurrence and progression rates were stated as $35.3 \%$ and $23.5 \%$ with a median follow-up of 41 months (7). In another cohort using a conductive heating system with 40 high-risk NMIBC patients, the recurrence rate was $32.5 \%$ and the median recurrence-free survival period was 23 months (8). In a randomised prospective study, the recurrence-free survival at the 24th month was $81.8 \%$ for TCT + MMC and $64.8 \%$ for the BCG arm. Progression was observed in one patient with BCG (1.4\%). In addition, in the case of $\mathrm{CIS}$, no statistically significant difference was noted between the BCG and TCT + MMC groups (6). Unlike in the studies in the literature, this study evaluated the intermediateand high-risk NMIBC groups separately. Initially, we planned to compare the TCT + MMC and BCG groups, but the unavailability of BCG did not allow it. The recurrence rates of 14 intermediate- 
risk and 30 high-risk patients in this study were $28.6 \%$ and $23.3 \%$, respectively. The progression rates were $7.1 \%, 23.3 \%$, respectively. As compared with the published series, the recurrence rate was better, although the progression rate was higher in the study group.

Considering the adverse events, MMC showed a better profile than BCG. In a meta-analysis comparing intravesical treatments, $27 \%-84 \%$ granulomatous cystitis or lower urinary tract symptoms (dysuria, urgency and frequency), 21\%-72\% macroscopic haematuria, and $27 \%-44 \%$ fever were reported as BCG side effects (10). In another study that compared MMC + TCT with BCG application, bladder spasm (14.4\%) and bladder pain $(14.1 \%)$ were noted as the main side effects in the MMC arm, whereas dysuria (11.7\%), nocturia (10.3\%), and frequency $(9.9 \%)$ were the side effects noted in the BCG arm. In this study, the sole adverse event was mild allergic reactions treated with oral agents and dysuria lasting for 2-3 days. This symptomatic process was treated conservatively. All the side effects recorded were of a grade $\leq 2$.

\section{Study Limitations}

The limitations of this study were the small sample size and the single-arm approach. To attain more powerful oncologic results, there is no doubt the need for randomized double arm studies with more patients comparing MMC + BWT and BCG.

\section{Conclusion}

Comparing our results with that of the literature, MMC with BWT had comparable recurrence and progression rates as BCG, with the number of side effects being significantly lower than those with BCG. Thus, MMC + BWT can be considered as an alternative treatment option for selected cases in whom BCG cannot be applied or is not available. Furthermore, MMC + BWT costs less and produces lesser side effects compared with other hyperthermia systems, making it an attractive option.

\section{Acknowledgements}

Publication: This study was presented in the $6^{\text {th }}$ Urooncology Winter Congress and Course on January 29- February 10 in 2015, Baku, Azerbaijan.

Contribution: There is not any contributors who may not be listed as authors.

Conflict of Interest: No conflict of interest was declared by the authors.

Financial Disclosure: The authors declared that this study received no financial support.

\section{Ethics}

Ethics Committee Approval: The study protocol was approved by the Erciyes University Clinical Research Ethics Committee (decision number: 2014/387, date: 04.07.2014).

Informed Consent: An informed consent was obtained from all the patients.

Peer-review: Externally peer-reviewed.

\section{Authorship Contributions}

Concept: G.S., A.D., Design: G.S., Ak.D., A.D., Data Collection and Processing: S..T.T., T.D., Ak.D., Analysis and Interpretation: A.D., A.T., T.D., Literature Search: Ş.T.T., Writing: A.D.

\section{References}

1. Ferlay J, Colombet M, Soerjomataram I, et al. Estimating the global cancer incidence and mortality in 2018: GLOBOCAN sources and methods. Int J Cancer 2019;144:1941-1953.

2. Burger M, Catto JW, Dalbagni G, et al. Epidemiology and risk factors of urothelial bladder cancer. Eur Urol 2013;63:234-241.

3. Babjuk M, Böhle A, Burger $M$, et al. EAU guidelines on non-muscleinvasive urothelial carcinoma of the bladder: update 2016. Eur Urol 2017;71:447-461.

4. Moskovitz B, Meyer G, Kravtzov A, et al. Thermo-chemotherapy for intermediate or high-risk recurrent superficial bladder cancer patients. Ann Oncol 2005;16:585-589.

5. Colombo R, Salonia A, Leib Z, et al. Long-term outcomes of a randomized controlled trial comparing thermochemotherapy with mitomycin- $C$ alone as adjuvant treatment for non-muscle-invasive bladder cancer (NMIBC). BJUI 2011;107:912-918.

6. Arends TJ, Nativ O, Maffezzini M, et al. Results of a randomised controlled trial comparing intravesical chemohyperthermia with mitomycin $C$ versus bacillus calmette-guérin for adjuvant treatment of patients with intermediate- and high-risk non-muscle-invasive bladder cancer. Eur Urol 2016;69:1046-1052.

7. Soria F, Milla P, Fiorito C, et al. Efficacy and safety of a new device for intravesical thermochemotherapy in non-grade 3 BCG recurrent NMIBC: a phase I-II study. World J Urol 2016;34:189-195.

8. Ekin RG, Akarken I, Cakmak O, et al. Results of intravesical chemohyperthermia in high-risk non-muscle invasive bladder cancer. Asian Pac J Cancer Prev 2015;16:3241-3245.

9. U.S. Department of Health and Human Services. Common Terminology Criteria for Adverse Events (CTCAE) Version 4.0. https://evs.nci.nih.gov/ftp1/CTCAE/CTCAE_4.03/Archive/ CTCAE_4.0_2009-05-29_QuickReference_8.5x11.pdf

10. Chou R, Selph S, Buckley DI, et al. Intravesical therapy for the treatment of non-muscle invasive bladder cancer: a systematic review and meta-analysis. J Urol 2017;197:1189-1199. 